\section{Durable, colour-stable hybrid ceramics in two layers and three types}

Whether aesthetic anterior restorations or inlays, onlays and posterior crowns with high colour and surface stability, Shofu Block HC can be used for almost all indications for modern CAD/CAM technology and machined wet or dry in all standard milling units.

Shofu has added two-layer blanks to its line of hybrid ceramics and fitted blocks with universal holding pins.

So Shofu Block HC, a high-performance CAD/CAM material, is now available in three types (Universal, Cerec and Ceramill) and as one- and two-layer blocks. Users may choose from various high and low-translucency shades and two enamel shades, designed to reliably meet every need in the field of all-ceramic restorations.

The excellent physical properties of Shofu Block HC allows making crowns and implant-supported restorations characterised by great durability and absorption of occlusal forces. Moreover, highly aesthetic restorations can be created thanks to enamel-like light transmission and the addition of two-layer blocks featuring a smooth, natural shade transition from dentine to enamel.

In combination with the HC Primer, which ensures very high bond strengths thanks to its unique infiltration effect, and SHOFU's gentle polishing and luting systems, a CAD/ CAM restoration system with perfectly matched components is provided - for all modern milling units, aesthetic requirements and clinical indications.

For further information, visit Shofu UK at the BDIA Dental Showcase 2018 at Stand D40 or call 01732783580 or email sales@shofu.co.uk.

\section{Tough on germs, gentle on equipment}

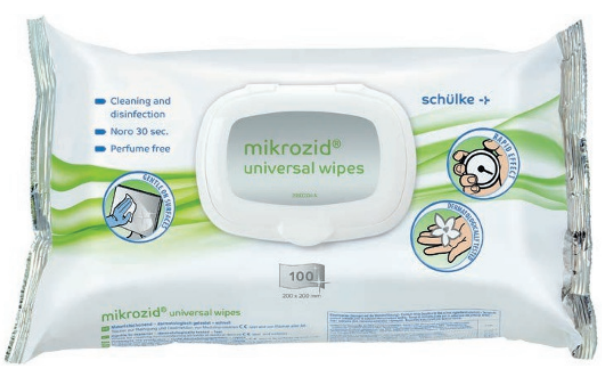

Effective cleaning of surfaces is essential to protect both staff and patients from the risk of cross infection. The mikrozid

range offers products for all areas of the dental practice.

Particularly popular is low-alcohol mikrozid universal which is effective against bacteria including $\mathrm{TB}$, as well as viruses like norovirus.

It offers dual cleaning and disinfection of surfaces, contains added surfactants to boost cleaning performance and is available in both liquid and wipes.

Mikrozid universal is fast acting and has excellent material compatibility. It can be used to clean smart phones, keyboards and other sensitive equipment, as well as dental chairs and surfaces.

To find out more about schülke's extensive infection prevention and control range, contact schülke UK on 01142543500 or visitwww.schuelke.com or email: mail.uk@schuelke.com.

\section{Discover the benefits of digital dentistry}

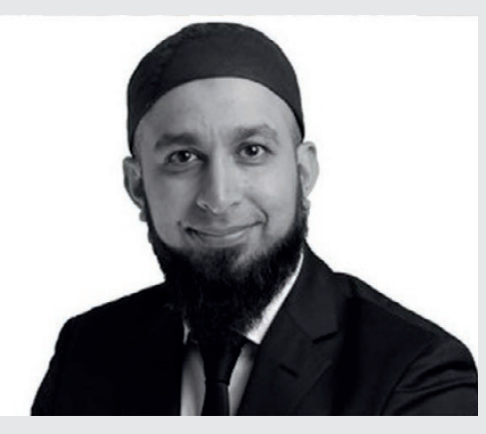

Dr Jameel Gardee is a certified Master and Instructor in Digital Smile Design (DSD), and is keen to share his knowledge at this year's British Academy of Cosmetic Dentistry

(BACD) Annual

Conference taking place in November in London,

where he will lead a session entitled 'Smile Rehab The Digital Way'.

Gardee says: 'I will show delegates how they can use digital technology to produce both $2 \mathrm{D}$ and $3 \mathrm{D}$ designs of beautiful smiles, allowing patients to see the final result before any procedures commence - thereby increasing treatment acceptance rates.

'I will then demonstrate how to deliver a predictable, final restoration using various systems from CEREC, Trios, Carestream and iTero. I hope delegates realise that digital dentistry can be just as effective, if not more so, than traditional dentistry.

'I'm definitely looking forward to attending the BACD Annual Conference. There will be a lot of great speakers in attendance; lots of fantastic hands-on workshops and delegates should also make the most of the various social events taking place. I would encourage everyone to book tickets early'.

Also at the conference, Dr Elaine Halley will be leading a session entitled 'Digital Smile Design: Welcoming a new era for orthodontic and restorative workflow'

Speaking about her upcoming presentation, Halley says: 'I will be

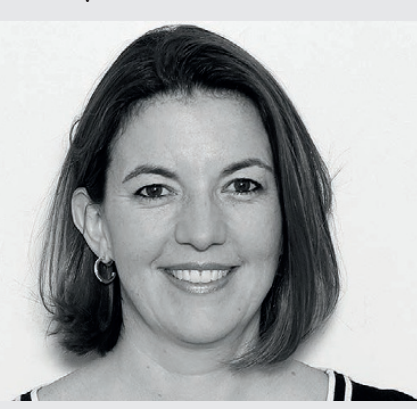

looking at the benefits of using the iTero scanner and how the DSDApp can be employed as a way of showing patients what their smile could look like post-treatment.

'I will also demonstrate how DSD can become part of the treatment planning process, enabling clinicians to produce more predictable results. Delegates should leave with a clear understanding of the step-by-step process involved with integrating faciallydriven treatment plans using digital technology'.

Dr Halley adds: 'The aim of the BACD has always been to bring the best dental education to members and non-members alike, thereby creating a community that is incredibly inclusive - regardless of experience or position within the profession. The excitement and enthusiasm for dentistry is evident in delegates that attend the Conference every year.'

The BACD Fifteenth Annual Conference 2018 entitled 'The Functional Smile: Start With Why...' takes place on 8-10 November 2018 at the Millennium Gloucester Hotel, London.

For more information, visit www.bacd.com. 